\title{
Freeform optics adapted to the design of spatial instrumentation for Earth observation
}

\section{Óptica Freeform adaptada al diseño de instrumentación espacial para observación de la Tierra}

\author{
A. García-Moreno*, L. M. González, T. Belenguer ${ }^{S}$ \\ Instituto Nacional de Técnica Aeroespacial (INTA), Área de Óptica Espacial, Torrejón de Ardoz, \\ Madrid, Spain

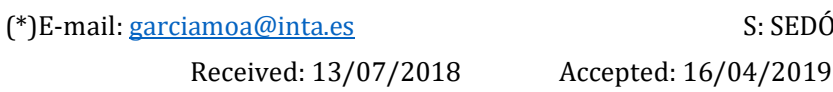 \\ DOI: $10.7149 /$ OPA.52.1.51006
}

\begin{abstract}
:
Freeform surfaces have been introduced in the optical design of a two-mirror Cassegrain telescope for Earth observation purposes. The application requires two field positions at the image plane with minimum aberrations where two micro spectrometers (HAMAMATSU C12880MA) shall be placed. These micro spectrometers are small and compact devices that incorporate the slit, a concave diffractive element and the CMOS sensor in the same component. Although these are very small components their position at the image plane are slightly deviated from the optical axis, where it is well known minimum aberrations occurs for classical optical systems. Freeform surfaces are introduced to correct those aberrations, mainly astigmatism and coma, for the specific fields determined by the positions of the micro spectrometers. Nodal Aberration Theory (NAT) will be used to determine the mirrors surface deformation as a set of $\phi$-polynomials calculating, at the same time, the effects of such deformations on the total aberrations
\end{abstract}

Key words: Freeform Optics, Spatial Instrumentation, Earth Observation, Nodal Aberration Theory (NAT)

\section{RESUMEN:}

Se han introducido superficies freeform en el diseño de un telescopio Cassegrain de dos espejos para observación de la Tierra. En el plano imagen de este telescopio, se colocarán 2 micro espectrómetros (HAMAMATSU C12880MA) en diferentes posiciones de dicho plano. Estos micro espectrómetros son pequeños y compactos dispositivos que integran la rendija de entrada, un elemento difractivo cóncavo y el sensor CMOS. La misión espacial requiere incluir al menos dos dispositivos en el plano focal. Aunque su tamaño ha sido considerablemente reducido, sus posiciones en el plano imagen generan una restricción en los campos que deben alejarse del eje óptico. Se sabe que un campo fuera del eje óptico introduce aberraciones en este tipo de telescopio, aberraciones que aumentan a medida que el campo se aleja del eje. Se introducen superficies freeform para corregir estas aberraciones, principalmente astigmatismo y coma, para los campos concretos que quedan determinados por las posiciones de los micro espectrómetros.

Por lo tanto, por la Teoría Nodal de Aberraciones (NAT), se introducen deformaciones en las superficies que componen el telescopio mediante un conjunto de polinomios con dependencia angular ( $\phi$-polynomials) y se calculan los efectos que dichas deformaciones tienen en las aberraciones totales en el plano imagen.

Palabras clave: Óptica Freeform, Instrumentación Espacial, Observación de la Tierra, Teoría Nodal de Aberraciones (NAT) 


\section{REFERENCES AND LINKS / REFERENCIAS Y ENLACES}

[1] J. Wang, B. Guo, Q. Sun, Z. Lu, "Third-order aberration fields of pupil decentered optical systems". Optics Express, 20(11), 11652-11658 (2012).

[2] K. P. Thompson, "Aberration fields in tilted and decentered optical systems". PhD, University of Arizona (1980).

[3] K. Fuerschbach, J. P. Rolland \& K. P. Thompson, "Extending Nodal Aberration Theory to include mount-induced aberrations with applications to freeform surfaces". Optics Express, 20(18), 20139-20155 (2012).

[4] K. Fuerschbach, J. P. Rolland \& K. P. Thompson, "Theory of aberration fields for general optical systems with freeform surfaces". Optics Express, 22(22), 26585-26606 (2014).

[5] K. Fuerschbach, "Freeform, $\varphi$-polynomial optical surfaces: optical design, fabrication and assembly". PhD, University of Rochester (2014).

[6] H. G. Rutten \& M. A. Van Venrooij, "Telescope optics: a comprehensive manual for amateur astronomers". Willmann-Bell (2002).

[7] The Fringe Zernike polynomial set was developed by John Loomis at University of Arizona in the 1970s. It is described in the Code V® (Synapsis, USA) Reference Manual.

[8] H. H. Hopkins, "The Wave Theory of Aberrations". Oxford on Clarendom Press, p. 48 (1998).

\section{Introduction}

Freeform optics is a powerful design option consisting in using optical surfaces in which rotational symmetry has been broken to achieve high quality in strongly aberrated optical systems. The design and fabrication of freeform optics provides a tool to recover high optical quality, for instance, in decentered or obscured optical systems, volume-restricted systems or systems with a large field of view.

The Nodal Aberration Theory (NAT) is the mathematical tool employed to study systems in which the rotational symmetry is not preserved. In principle, this theory was limited to optical systems with rotationally symmetric surfaces that were tilted or decentered $[1,2]$. However, the emergence of freeform optics stimulated the extension of NAT to systems including non-rotationally symmetric components $[3,4,5]$.

NAT states that non-rotationally symmetric surfaces in an optical system do not originate any new kind of aberrations but a new distribution of the optical aberrations at the image plane in terms of the field, in other words, a new distribution of the field aberrations $[3,4,5]$. This lack of symmetry produces the appearance of one or more nodes of aberrations (zero or almost zero aberration field points) away from the optical axis. Hence, a proper lack of rotational symmetry allows the optical designer selecting the position of the nodes of aberrations at the image plane.

NAT also stablishes that a freeform surface coincident with the aperture stop generates constant aberrations at the image field. However, as that surface is moved axially apart from the aperture stop, the aberrations at the image plane start taking a field dependency. Now, aberrations depend of the coordinates of the displaced pupil: $W(\vec{\rho}) \rightarrow W(\vec{\rho}+\Delta \vec{h})$, where $\rho$ is the pupil normalized radius and $\Delta \vec{h}$ the freeform surface displacement with respect to the pupil generating off-axis aberration nodes. Therefore, NAT allows calculating the final contribution to aberrations when a non-rotationally symmetric surface displaced from the stop is introduced in the system. $[3,4,5]$.

Objective of this paper is presenting the use of freeform optics in a spaxce instrument, specifically a Cassegrain telescope for Earth Observation purposes. Space optics is very conservative due to complexity in design, fabrication and verification of typically large aperture elements, lenses or mirrors. In this sense, it is very unusual to use freeform surfaces and so, take advantage of its potential in terms of performances improvement. In this paper, we start from a rotationally symmetric Cassegrain telescope with two micro spectrometers (HAMAMATSU C12880MA) placed at the image plane. The mission requires two simultaneous observations at different positions in the along-track direction. Sensor size causes a restriction in field magnitudes taken away from the optical axis. Cassegrain telescopes present high quality at very low fields but aberrations increase as the field 
does [6]. Therefore, at the selected field positions, the system presents high aberrations, mainly astigmatism and coma. Therefore, we must appeal to freeform optics to match the nodes of aberrations with the fields selected by the positions of the micro spectrometers.

We firstly present the analytical minimization of astigmatism and coma at the fields of interest following strictly the NAT theory, and secondly the modelling validation using an optical design tool like Code V® (Synapsis, USA). Ultimately, we show the resulting freeform surfaces of the two mirrors comprising the system.

\section{Initial design: Rotationally symmetric design}

A Cassegrain telescope is typically composed by a primary concave mirror and a secondary convex mirror, both of them aligned along the optical axis, so the secondary mirror generates an obscuration on the primary one. As starting point, we have considered two conic mirrors with small asphericities and the entrance pupil in front of the primary mirror, approximately in the same plane where the secondary mirror is. Because of volume restrictions, the primary-secondary distance is limited to be shorter than $55 \mathrm{~mm}$. In addition, the sensors and image plane shall be placed just behind the primary mirror. This is forcing the design to be very compact and reducing the degrees of freedom in the optical design to achieve a well-optimized system.

The instrument shall orbit at a height of $450 \mathrm{Km}$, the required GSD (Ground Sample Distance, projection on ground of the spatial resolving element) is $60 \mathrm{~m}$ and the pixel size at the image plane is $50 \mu \mathrm{m} \times 50 \mu \mathrm{m}$. Therefore, the value of the Effective Focal Length (EFL) is $375 \mathrm{~mm}$. The telescope envelope is limited by the CubeSat frame were it shall be accommodated, a $100 \mathrm{~mm}$-side cube, limiting the Entrance Pupil Diameter (EPD) to $80 \mathrm{~mm}$ and the maximum total length of the system to $65 \mathrm{~mm}$. Numerical Aperture (NA) is so equivalent to 0.11 .

The fields selected by the positions of the micro spectrometers in the dimensions of the image height are $F_{1}=(-0.05,13.00) \mathrm{mm}$ and $F_{2}=(0.05,-13.00) \mathrm{mm}$, corresponding to the object fields of $F_{1}=$ $\left(-0.0085^{\circ}, 2.22^{\circ}\right)$ and $F_{2}=\left(0.0085^{\circ},-2.22^{\circ}\right)$. As we can see, these angular values exceed by far the typical values for this type of telescopes $\left( \pm 1.5^{\circ}\right)$ and, as consequence, high aberrations are expected at these positions [6].

\section{Break of the rotational symmetry}

\section{3. a. Set of $\phi$-polynomials: Fringe Zernike polynomials}

In order to introduce the lack of rotational symmetry on the optical surfaces, we shall define the surface deformation in terms of a set of $\varphi$-polynomials not only depending on the radial component, also in angular. The surface sagitta can be expressed by equation 1 where the first term represents the conic base and the second term is a fifth order Zernike polynomials expansion according to Fringe basis [7].

$$
\operatorname{Sag}_{M_{1}, M_{2}}=\frac{C_{M_{1}, M_{2}} r^{2}}{1+\sqrt{1-\left(1+K_{M_{1}, M_{2}}\right) C_{M_{1}, M_{2}}^{2} r^{2}}}+\sum_{i=1}^{16} Z_{i_{M_{1}, M_{2}}} P_{i_{M_{1}, M_{2}}}(\rho, \varphi)
$$

In equation $1, C_{M_{1}, M_{2}}$ is the curvature of each mirror, $K_{M_{1}, M_{2}}$ the conic constant, $r$ the radial component, $Z_{i}$ the Zernike coefficients, and $P_{i}(\rho, \varphi)$ the Zernike polynomials that depend of the normalized radius $(\rho)$ and the angular component $(\varphi)$. From equation 1 , we can observe the two contributions to the sagitta of each mirror.

In our case, the candidates to be introduced in the figuring of the mirrors with the purpose of breaking rotational symmetry will be the Zernike polynomials that break this concrete symmetry. This excludes Zernike polynomials corresponding to the aberrations of piston $\left(Z_{1}\right)$, focus $\left(Z_{4}\right)$, spherical aberration $\left(Z_{9}\right)$ and fifth order spherical aberration $\left(Z_{16}\right)$. We will also exclude tilt Zernike polynomials $\left(Z_{2}\right.$ and $\left.Z_{3}\right)$ because they can by achieve just by tilting mirrors. Hence, the Zernike polynomials to be considered in the mirrors surface deformations are: astigmatism $\left(\mathrm{Z}_{5}\right.$ and $\left.\mathrm{Z}_{6}\right)$, coma $\left(\mathrm{Z}_{7}\right.$ and $\left.\mathrm{Z}_{8}\right)$, trefoil $\left(\mathrm{Z}_{10}\right.$ and $\left.\mathrm{Z}_{11}\right)$, secondary astigmatism $\left(\mathrm{Z}_{12}\right.$ and $\left.\mathrm{Z}_{13}\right)$ and secondary coma $\left(\mathrm{Z}_{14}\right.$ and $\left.\mathrm{Z}_{15}\right)$. 


\section{3. b. Effect of the Zernike polynomials on the aberrations at the image plane}

As said before, NAT allows calculating the contribution of introducing each Zernike polynomial as a deformation on a surface displaced from the stop. Aberrations have been calculated in terms of the coordinates of the displaced pupil: $W(\vec{\rho}) \rightarrow W(\vec{\rho}+\Delta \vec{h})$ where $\Delta \vec{h}$ is the pupil displacement for different values of the field.

Assuming that $y$ is the marginal ray height, $\bar{y}$ the chief ray height on the surface in which the symmetry is broken and $\vec{H}$ a vector including the normalized field coordinates, we have

$$
\Delta \vec{h}=\left(\frac{\bar{y}}{y}\right) \vec{H}
$$

Introducing this displacement in the expression for the total aberrations as a Zernike polynomials series expansion according to the wavefront theory of aberrations [8], we can obtain the effects produced by the deformation of the surfaces comprising the optical system. The deduction of the effects produced by the surfaces deformation can be found in $[4,5]$. According to this and to the type of aberrations present on the initial design we will assume that the Zernike coefficients of astigmatism $\left(\mathrm{Z}_{5}\right.$ and $\left.\mathrm{Z}_{6}\right)$ and secondary astigmatism $\left(\mathrm{Z}_{12}\right.$ and $\left.\mathrm{Z}_{13}\right)$ are suitable to be introduced in the sagitta of the mirrors for minimizing analytically the total aberrations of astigmatism and coma at the fields of interest.

Specifically, the contribution to the total aberrations at the image plane due to the presence of astigmatism and secondary astigmatism on the mirrors topography are presented in equations 3 and $4[4,5]$.

$$
\begin{aligned}
W_{Z_{5}, Z_{6}}^{F F}= & \frac{1}{2}(\underbrace{\vec{B}_{222}^{2} \cdot \vec{\rho}^{2}}_{\begin{array}{c}
\text { Field independant } \\
\text { astigmatism }
\end{array}}+2 \vec{B}_{222}^{2} \Delta \vec{h}^{*} \cdot \vec{\rho}+\vec{B}_{222}^{2} \cdot \Delta \vec{h}^{2}) \\
W_{Z_{12}, Z_{13}}^{F F}=\frac{1}{2}( & \left(\vec{B}_{242}^{2} \cdot \vec{\rho}^{2}\right)(\vec{\rho} \cdot \vec{\rho})+\underbrace{3\left(\vec{B}_{242}^{2} \Delta \vec{h}^{*} \cdot \vec{\rho}\right)\left(\vec{\rho} \cdot \overrightarrow{B^{2}}\right)}_{\text {Field dependant coma }}+\vec{B}_{242}^{2} \Delta \vec{h} \cdot \vec{\rho}^{3} \\
& +\underbrace{3(\Delta \vec{h} \cdot \Delta \vec{h})\left(\vec{B}_{242}^{2} \cdot \vec{\rho}^{2}\right)}_{\text {Field dependant astigmatism }}+3\left(\vec{B}_{242}^{2} \cdot \Delta \vec{h}^{2}\right)(\vec{\rho} \cdot \vec{\rho}) \\
& +2\left(\vec{B}_{242}^{2} \cdot \Delta \vec{h}^{2}\right)(\Delta \vec{h} \cdot \vec{\rho})+2(\Delta \vec{h} \cdot \Delta \vec{h})\left(\vec{B}_{242}^{2} \Delta \vec{h}^{*} \cdot \vec{\rho}\right) \\
& \left.+(\Delta \vec{h} \cdot \Delta \vec{h})\left(\vec{B}_{242}^{2} \cdot \Delta \vec{h}^{2}\right)\right)
\end{aligned}
$$

In equation $3, W_{Z_{5}, Z_{6}}^{F F}$ represents the total amount of aberrations at the image plane generated when the Zernike polynomials of astigmatism (coefficients $\mathrm{Z}_{5}$ and $\mathrm{Z}_{6}$ ) are introduced in the mirrors topography. $\vec{B}_{222}^{2}$ is a vector representing the surface astigmatism magnitude and orientation and shall be calculated in order to minimize aberrations at the field points of interest. We observed that this astigmatism contributes to the total aberrations with a term of constant astigmatism in field and two other terms, piston and tilt.

Similarly, in equation $4, W_{Z_{12}, Z_{13}}^{F F}$ represents the total aberrations at the image plane generated when the Zernike polynomials of secondary astigmatism $\left(\mathrm{Z}_{12}\right.$ and $\left.\mathrm{Z}_{13}\right)$ are introduced in the mirrors topography. $\vec{B}_{242}^{2}$ is also a vector including the surface secondary astigmatism magnitude and orientation and shall be calculated again to minimize aberrations at the desired points. Terms in equation 4 can be interpreted as follows: first term is a secondary astigmatism term and the unique field independent, second term is the coma contribution, third term is trefoil, fourth term is astigmatism and the fifth one is a field curvature contribution. The rest of the terms does not produce an image deformation, so they are not taken into account. The only terms considered in the analytical calculation are the terms corresponding to astigmatism and coma because those are the aberrations we want to minimize.

According to the type of aberrations present on the initial design and the potential corrector effect on the total aberrations provided by the mirrors deformation given by the Zernike astigmatism $\left(\mathrm{Z}_{5}\right.$ 
and $\mathrm{Z}_{6}$ ) and secondary astigmatism $\left(\mathrm{Z}_{12}\right.$ and $\left.\mathrm{Z}_{13}\right)$, we will try to find analytically the mirror topography defined by $\vec{B}_{222}^{2}$ and $\vec{B}_{242}^{2}$ that minimizes astigmatism and coma at the fields of interest.

\section{Freeform design: analytical model}

Basically, the idea is to find the astigmatism and secondary astigmatism magnitude and orientation of the mirror topography to minimize the astigmatism and coma aberrations at the image plane at two off-axis fields given by $\vec{H}_{1}$ and $\vec{H}_{2}=-\vec{H}_{1}$. We shall find the magnitude and orientation of the vectors $\vec{B}_{222}^{2}$ and $\vec{B}_{242}^{2}$. For this purpose, we will take advantage of the representation of vectors in complex space: $\vec{H}_{1}=H_{1} e^{i \theta_{1}}, \vec{B}_{242}^{2}=B_{242}^{2} e^{i 2 \varphi_{242}}$ and $\vec{B}_{222}^{2}=B_{222}^{2} e^{i 2 \varphi_{222}}$

The total astigmatism is the sum of the initial design rotationally symmetric contribution given by $W_{222}$ [8] and freeform deformations contribution coming from the astigmatism and secondary astigmatism given by $\vec{B}_{222}^{2}$ and $\vec{B}_{242}^{2}$ as expressed in equations 3 and 4 . Thus, the total astigmatism aberration at the image plane is given by

$$
W_{A s t}=\frac{1}{2}\left(W_{222} \vec{H}^{2}+\vec{B}_{222}^{2}+3 \vec{B}_{242}^{2} \vec{H}^{2}\right) \vec{\rho}^{2}
$$

With respect to coma, there is also a contribution from the initial design given by $W_{131}$ [8] and a contribution from the freeform deformations due to the secondary astigmatism. This contribution is extracted from equation 4 . Thus, the total coma aberration at the image plane is given by

$$
W_{\text {Coma }}=\left(W_{131} \vec{H}+\frac{3}{2} \vec{B}_{242}^{2} \vec{H}^{*}\right) \vec{\rho}(\vec{\rho} \cdot \vec{\rho})
$$

Therefore, to place the nodes of these aberrations at $\vec{H}_{1}$ and $\vec{H}_{2}=-\vec{H}_{1}$, we have to solve the system of equations 7 and 8.

$$
\begin{gathered}
W_{222} \vec{H}_{1}^{2}+\vec{B}_{222}^{2}+3 \vec{B}_{242}^{2} \vec{H}_{1}^{2}=\overrightarrow{0} \\
W_{131} \vec{H}_{1}+\frac{3}{2} \vec{B}_{242}^{2} \vec{H}_{1}^{*}=\overrightarrow{0}
\end{gathered}
$$

Where $\mathrm{H}_{1}{ }^{*}$ means complex conjugated. Multiplying equation 8 by $\frac{\vec{H} \cdot \vec{H}^{*}}{|\vec{H}|}=1$ we obtain:

$$
\vec{H}_{1}^{*}\left(W_{131} \frac{\vec{H}_{1}^{2}}{\left|\vec{H}_{1}\right|^{2}}+\frac{3}{2} \vec{B}_{242}^{2}\right)=\overrightarrow{0}
$$

From equation 9, we can observe that the first node of coma is produced at $\vec{H}=\overrightarrow{0}$ (not coma on-axis as expected) and other nodes come from solving equation 10.

$$
W_{131} \frac{\vec{H}_{1}^{2}}{\left|\vec{H}_{1}\right|^{2}}+\frac{3}{2} \vec{B}_{242}^{2}=\overrightarrow{0}
$$

Writing each vector in its complex form:

$$
\begin{gathered}
\vec{B}_{242}^{2}=B_{242}^{2} e^{i 2 \varphi_{242}} \\
\vec{H}_{1}=H_{1} e^{i \theta_{1}} \rightarrow \frac{\vec{H}_{1}^{2}}{\left|\vec{H}_{1}\right|^{2}}=e^{i 2 \theta_{1}}
\end{gathered}
$$

and replacing on equation 10 yields:

$$
W_{131} e^{i 2 \theta_{1}}+\frac{3}{2} B_{242}^{2} e^{i 2 \varphi_{242}}=\overrightarrow{0}
$$

The solution for the magnitude and orientation of vector $\vec{B}_{242}^{2}$ is:

$$
\begin{gathered}
B_{242}^{2}=-\frac{2}{3} W_{131} \\
2 \varphi_{242}=2 \theta_{1}
\end{gathered}
$$


Then, we go back to equation 8 to calculate the magnitude and orientation of the vector $\vec{B}_{222}^{2}=$ $B_{222}^{2} e^{i 2 \varphi_{222}}$. Substituding on equation 8 the results obtained for $\vec{B}_{242}^{2}$ (eqs. 14 and 15), we get the following expression:

$$
W_{222} H_{1}^{2} e^{i 2 \theta_{1}}+B_{222}^{2} e^{i 2 \varphi_{222}}+3\left(-\frac{2}{3} W_{131} e^{i 2 \theta_{1}}\right) H_{1}^{2} e^{i 2 \theta_{1}}=\overrightarrow{0}
$$

And operating on equation 16, we obtain:

$$
B_{222}^{2} e^{i 2 \varphi_{222}}=\left(2 W_{131} e^{i 4 \theta_{1}}-W_{222} e^{i 2 \theta_{1}}\right) H_{1}^{2}
$$

Therefore, the solution for the magnitude and orientation for vector $\vec{B}_{222}^{2}$ is:

$$
\begin{aligned}
& B_{222}^{2}=H_{1}^{2} \sqrt{W_{222}^{2}+4 W_{131}^{2}-4 W_{222} W_{131} \cos \left(2 \theta_{1}\right)} \\
& 2 \varphi_{222}=\tan ^{-1}\left(\frac{2 W_{131} \sin \left(4 \theta_{1}\right)-W_{222} \sin \left(2 \theta_{1}\right)}{2 W_{131} \cos \left(4 \theta_{1}\right)-W_{222} \cos \left(2 \theta_{1}\right)}\right)
\end{aligned}
$$

So far, we have calculated the magnitude and orientation of the astigmatism (eqs. 18 and 19) and secondary astigmatism (eqs. 14 and 15) to place the nodes of aberrations at the fields selected by the positions of the micro spectrometers.

At this time, it should be noted that the vector $\vec{B}_{242}^{2}$, secondary astigmatism indicator that turns into coma corrector, does not depend on the field magnitude but the orientation. This means that coma aberration will not present different nodes at different points of the field. Instead, coma will present a node along the direction defined by the two target fields. Nevertheless, astigmatism, with a distribution at the image plane controlled by $\vec{B}_{222}^{2}$ and $\vec{B}_{242}^{2}$, will present two nodes at the two target fields. Following figures show both aberrations at the image plane, astigmatism (fig. 1(a)) and coma (fig. 1(b)). The RMS (root-mean-square) of the wavefront error has also been represented graphically (figure 2) as a figure of merit of the telescope optical quality, it measures the wavefront departure from the ideal reference sphere at the exit pupil.

As can be observed the use of freeform surfaces gets the two nodes to be coincident with the fields of interest where the two sensors should be placed.

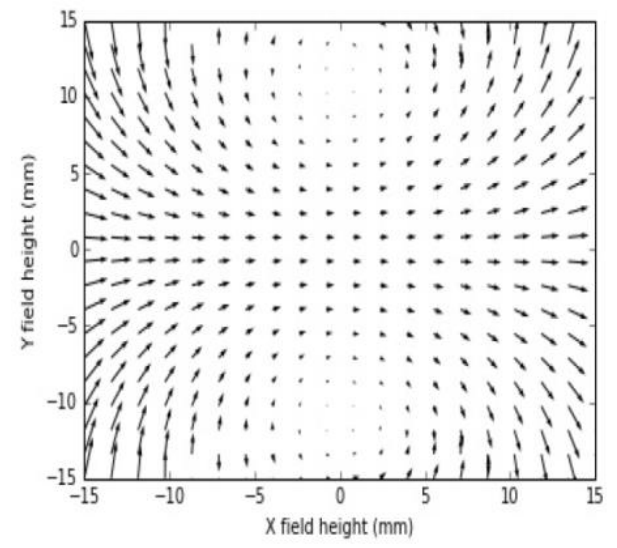

(a)

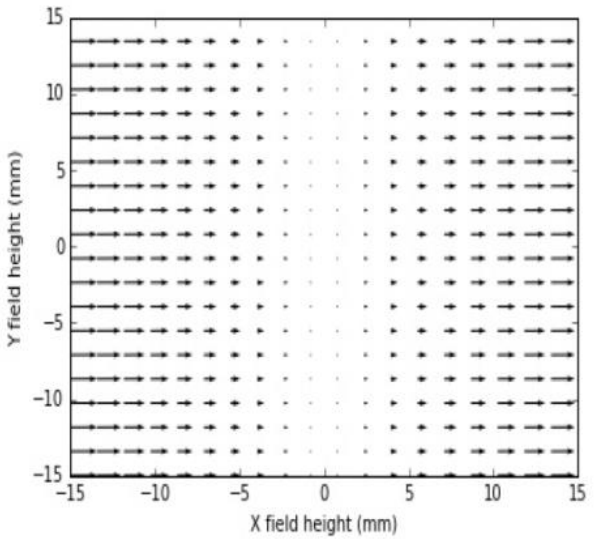

(b)

Fig 1. Aberrations distribution at the image plane after using freeform surfaces for the two mirrors. (a) Astigmatism. (b) Coma. 


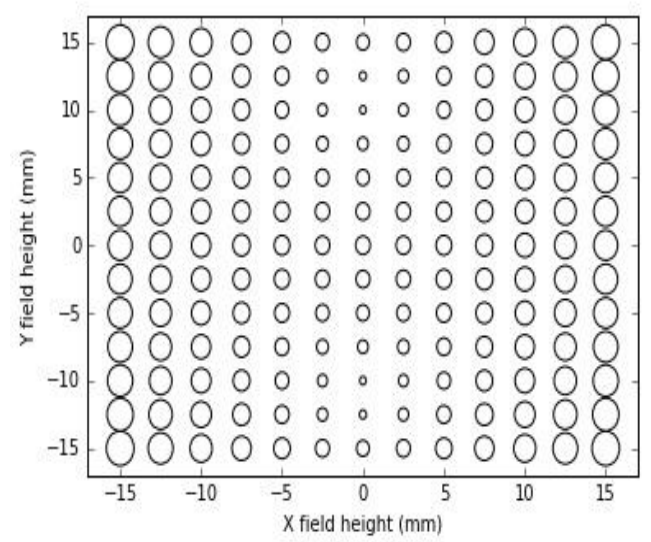

Fig 2. Distribution of the RMS at the image plane showing the two nodes of aberrations at non-zero fields.

\section{Freeform design: Code V optical design}

\section{5. a. Optical design}

Starting from the initial design presented in section 2, we optimize the freeform design using Code V. Defining both mirrors as Fringe Zernike polynomial surfaces and letting variable the Zernike coefficients corresponding to astigmatism $\left(\mathrm{Z}_{5}\right.$ and $\left.\mathrm{Z}_{6}\right)$ and secondary astigmatism $\left(\mathrm{Z}_{12}\right.$ and $\left.\mathrm{Z}_{13}\right)$, we optimize the freeform design. Table 1 shows the resulting values for the primary and secondary mirrors geometrical parameters. Optical solution is presented in figure 3.

Table 1. Parameters values for the freeform design.

\begin{tabular}{ccc} 
& $\mathbf{M}_{\mathbf{1}}$ & $\mathbf{M}_{\mathbf{2}}$ \\
\hline $\mathbf{R}(\mathbf{m m})$ & -121.8240 & -24.8624 \\
$\mathbf{K}$ & -1.0210 & -2.1804 \\
$\mathbf{Z}_{\mathbf{5}}(\boldsymbol{\mu \mathbf { m } )}$ & 9.2939 & 25.5668 \\
$\mathbf{Z}_{\mathbf{6}}(\boldsymbol{\mu \mathbf { m } )}$ & $7.706210^{-2}$ & 0.2059 \\
$\mathbf{Z}_{\mathbf{1 2}}(\boldsymbol{\mu \mathbf { m } )}$ & -0.2068 & 0.7633 \\
$\mathbf{Z}_{\mathbf{1 3}}(\boldsymbol{\mu \mathbf { m }})$ & $-1.578110^{-3}$ & $-6.056310^{-3}$
\end{tabular}

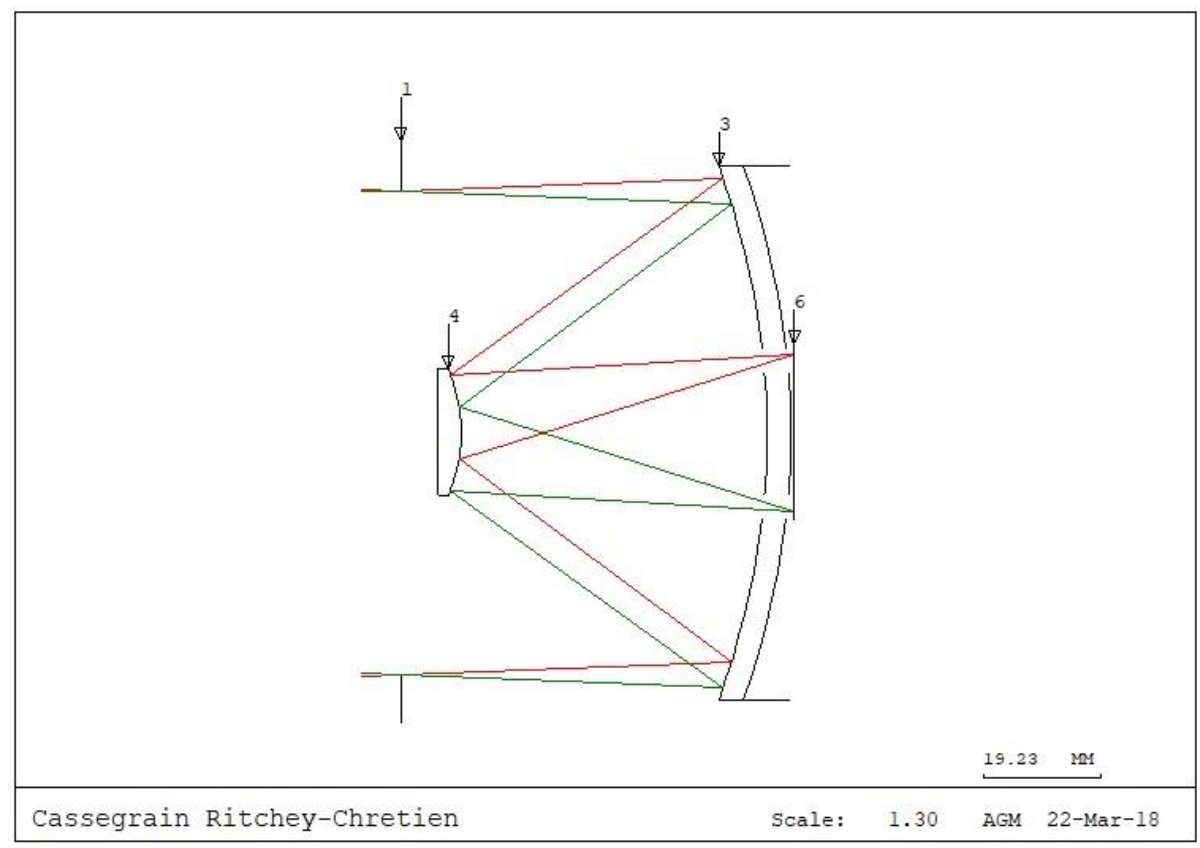

Fig 3. Optimized freeform design. Both mirrors break rotational symmetry on their figuring. 
Figure 4 shows the spot diagram for both, the initial systems that preserves rotational symmetry (Fig. 4a) and for the freeform system (Fig. 4b). It clearly shows how the optical quality improves in the system non-rotationally symmetric.

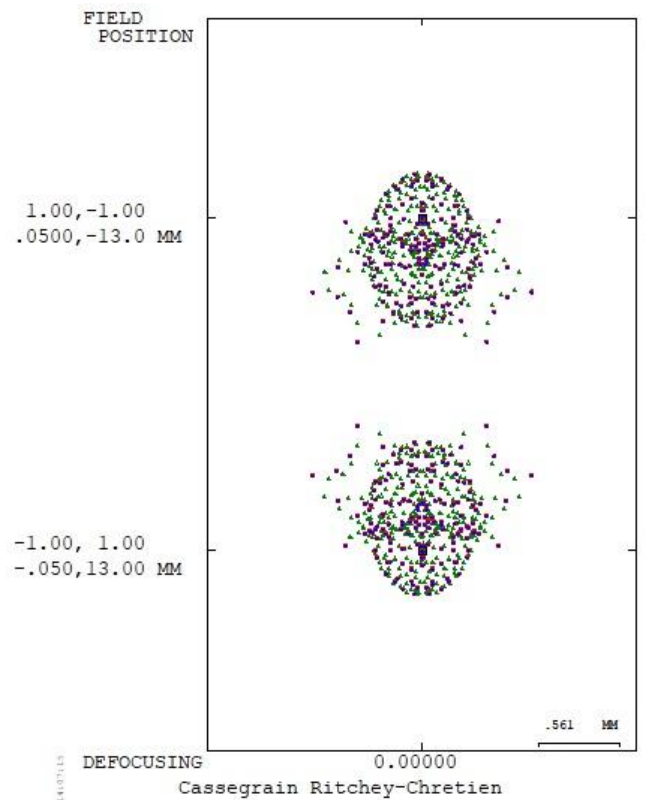

(a)

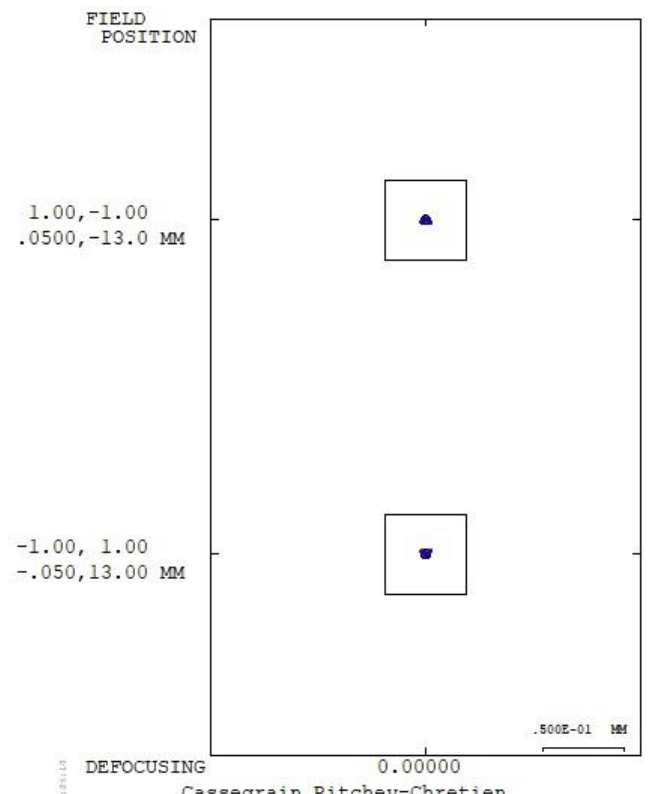

(b)

Fig 4. Spot diagram of the initial design presented in section 2 (a) in compare to the spot diagram for the freeform design (b) at the image plane. Square box represents the detector size (50 microns).

The MTF for the initial design is represented in the Figure 5 (a) while the MTF for the freeform design is shown in Figure 5 (b). The low optical quality obtained in the initial design is corrected with the freeform geometry. The freeform design is limited by diffraction, it means that it is well corrected for aberrations for the specific fields taken into account.

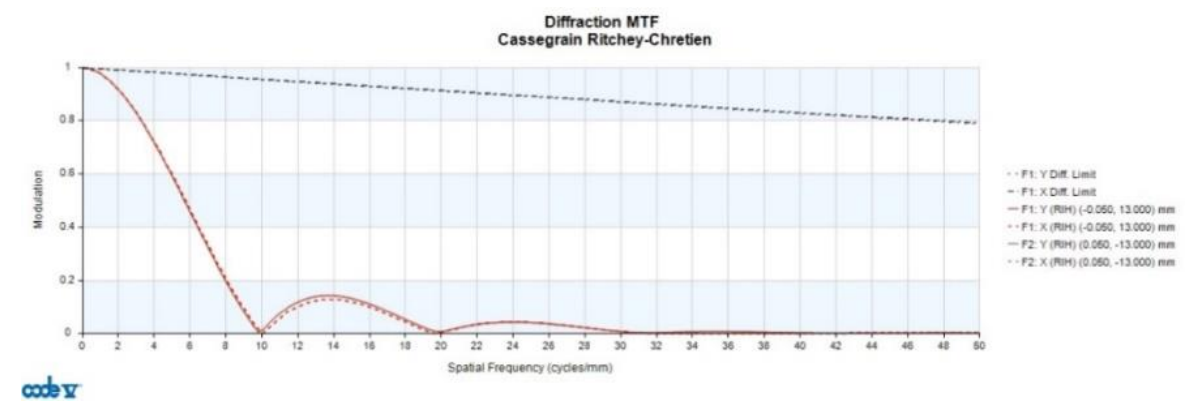

(a)

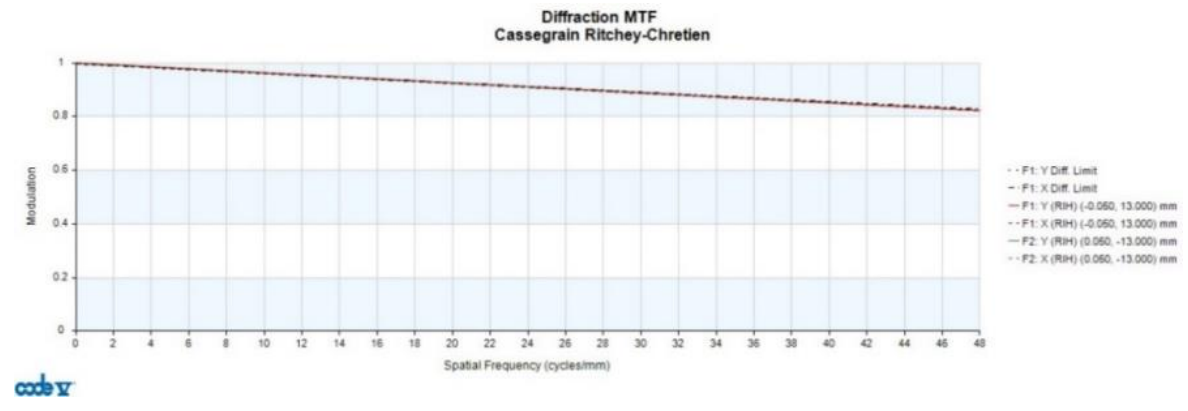

(b)

Fig 5. MTF for the initial design (a) in compare to the MTF for the freeform design (b). The black dashed line represents the diffraction limit. 
Figure 6 and 7 show the distribution of the aberrations of astigmatism and coma respectively at the image plane in terms of the real image height field. In both figures, the system with and without rotational symmetry are compared. Figures 6 (a) and 7 (a) show how the aberrations get their minimum value at the optical axis in the symmetric design while, in the freeform design, two nodes of aberrations show up at the field points previously selected (Figs. 6 (b) and 7 (b)).

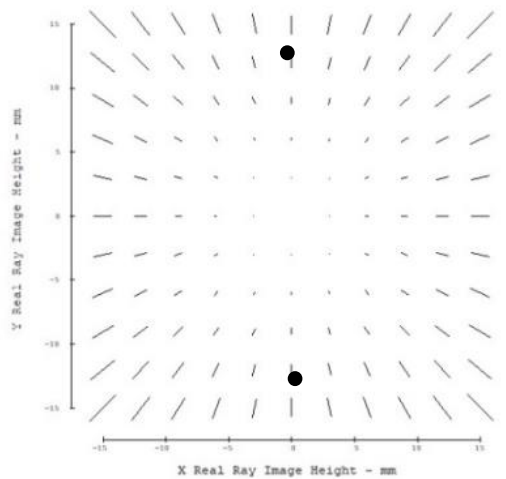

(a)

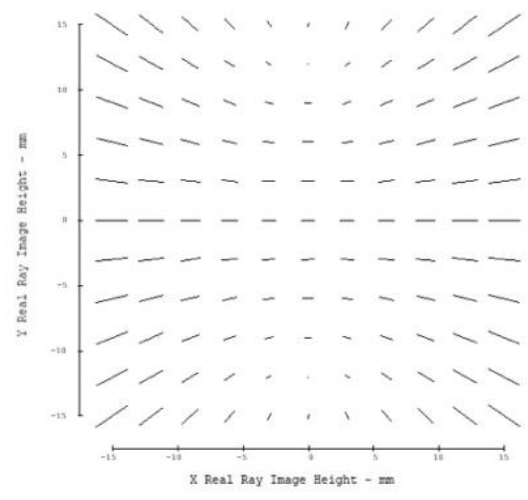

(b)

Fig 6. (a) Distribution of the astigmatism in terms of the real image height field for the rotationally symmetric initial design. (b) Distribution of the astigmatism in terms of the real image height field for the freeform design. Black dot in (a) represents the position of the fields of interest.

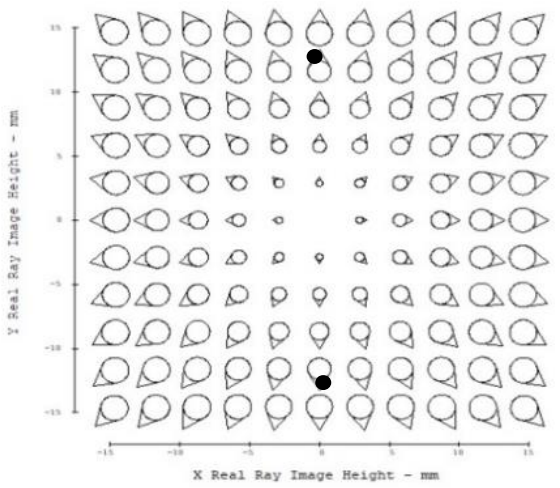

(a)

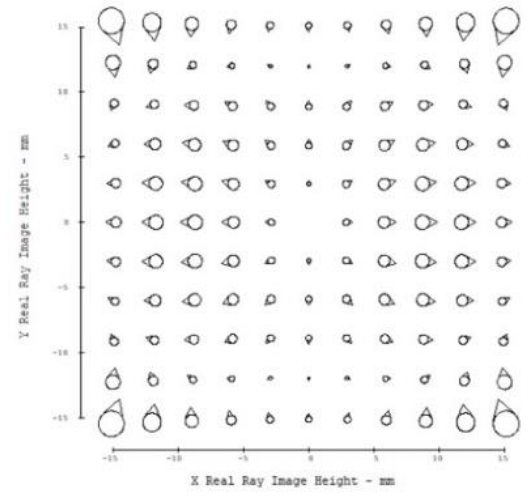

(b)

Fig 7. (a) Distribution of the coma aberration in terms of the real image height field for the rotationally symmetric initial design. (b) Distribution of the coma aberration in terms of the real image height field for the freeform design. Black dot in (a) represents the position of the fields of interest.

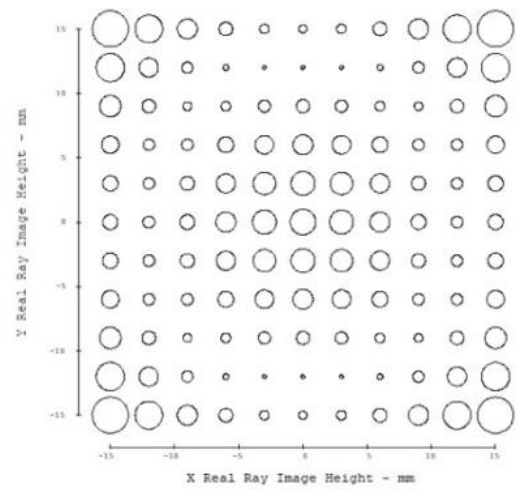

Fig 8. RMS in terms of the real image height field for the freeform design. 
Figure 8 shows the RMS for the freeform design at the image plane. Again, the RMS field distribution exhibits the nodes of aberrations.

As conclusion, both the analytical model (figures 1 and 2) and the CodeV design (figures 6(b), 7(b) and 8) demonstrate that using freeform surfaces the highest optical performances are achieved at the desired field positions.

\section{5. b. Mirrors surfaces}

Extracting the Zernike coefficients achieved to optimize the freeform design, we plot the sagitta of each mirror in order to know how its final topography is. Figures 9 and 10 represent the primary and secondary mirrors sagitta respectively. For each figure, the topography has been divided as the sum of the base conic (a) and the freeform contribution given by the Zernike expansion (b). As can be observed through the bar scales, the contribution of the base conic dominates over the Zernike polynomials contribution in both mirrors. Very small changes in mirrors figuring have a great impact in optical performances.

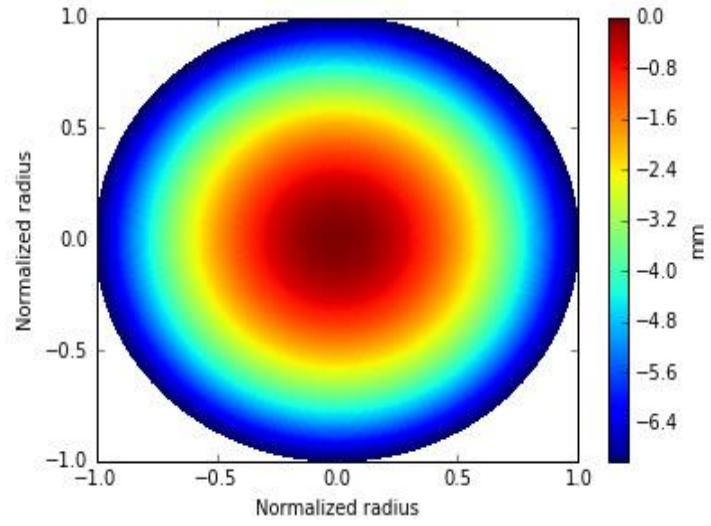

(a)

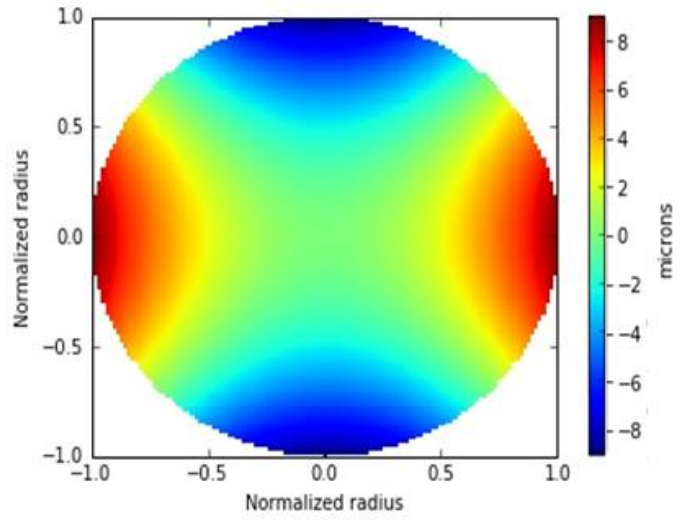

(b)

Fig 9. Sag of the primary mirror $\mathrm{M}_{1}$. (a) Base conic term for $\mathrm{M}_{1}$. (b) Freeform term given by Zernike polynomial expansion for $\mathrm{M}_{1}$.

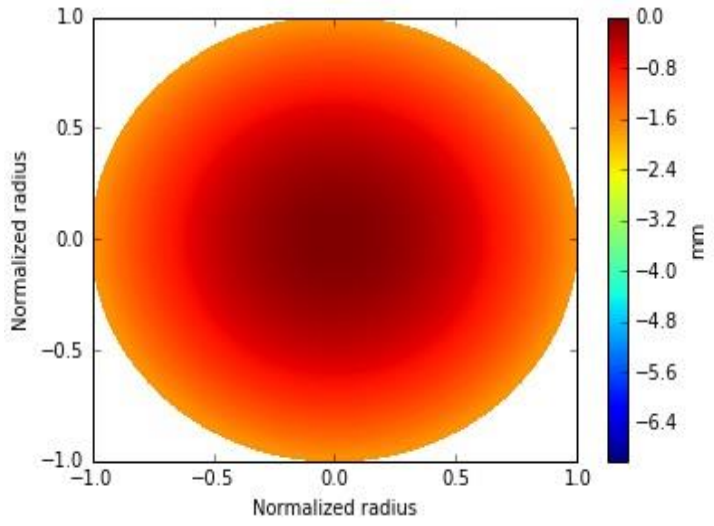

(a)

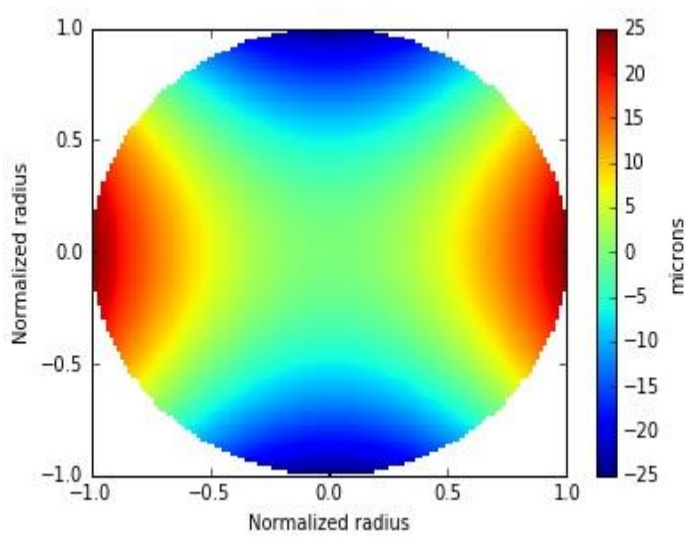

(b)

Fig 10. . Sag of the secondary mirror $\mathrm{M}_{2 .}$ (a) Base conic term for $\mathrm{M}_{2 .}$. (b) Freeform term given by Zernike polynomial expansion for $\mathrm{M}_{2}$. For clarity, scales for the graphs corresponding to the freeform contribution are different (right ones).

It is remarkable that figures $9(\mathrm{a})$ and 10 (a) share the same scale, so the base conic deformation is stronger in the primary mirror. Figures $9(\mathrm{~b})$ and $10(\mathrm{~b})$ do not share the same scale and it can be noticed that the deformation due to the Fringe Zernike polynomials, the deformation that breaks the rotational symmetry, is stronger in the secondary mirror. 


\section{Conclusions}

We have achieved a high quality optical system consisting in a two-mirror Cassegrain telescope for an angular field of view larger than $4^{\circ}$ using freeform surfaces for the two mirrors, freeform surfaces have been defined as a set of Zernike polynomials. To accomplish this high quality in systems preserving rotational symmetry, the use of at least three mirrors should be required, but freeform optics allows us to maintain the two-mirror concept.

In addition, we have only used astigmatism, primary and secondary, as source of mirrors deformation, avoiding the use of higher orders and so complexity in the fabrication and verification process. The freeform potential lies in the large capability to select Zernike orders. Evidently, the lower the order the simpler the solution and the manufacturing process, as has been the case presented here. In order to fabricate the components of this freeform optical systems, a tolerance analysis is performed. The resulting tolerance in the sagitta of the freeform mirrors is in the order of tens of nanometers, compatible with the typical mechanical shops capabilities.

In conclusion, using the Nodal Aberration Theory (NAT), we have developed an analytical model to describe the distribution of the total aberrations at the image plane along the field of view placing the emerging nodes at the field positions of interest. This analytical model agrees with the optical design of the telescope implemented using Code V.

\section{Acknowledgments}

Portions of this work were presented at the XII Reunión Nacional de Óptica as a poster named "Freeform optics adapted to the design of spatial instrumentation for Earth observation" (2018). 